\title{
Segregation and Integration of Visual Channels: Layer-by-Layer Computation of ON-OFF Signals by Amacrine Cell Dendrites
}

\author{
Ji-Jie Pang, Fan Gao, and Samuel M. Wu \\ Cullen Eye Institute, Baylor College of Medicine, Houston, Texas 77030
}

The visual system analyzes images through parallel channels, and our data suggest that the first set of parallel representations of the visual world is embodied in the inner plexiform layer (IPL) of the retina, in which light-evoked excitatory inputs of the ON and OFF bipolar cells to amacrine cells (ACs) are organized in a layer-by-layer manner. Approximately $30 \%$ of ACs have narrowly monostratified dendrites in 1 of the 10 strata of the $\mathrm{IPL}$, and they receive segregated bipolar cell inputs: the lightevoked excitatory cation current, $\Delta \mathrm{I}_{\mathrm{C}}$, in strata 1,2 , and 4 is OFF (predominantly mediated by the OFF bipolar cells), the current in strata 3 and $7-10$ is ON (predominantly mediated by ON bipolar cells), and the current in strata 5 and 6 is ON-OFF (mediated by both ON and OFF bipolar cells). The remaining $70 \%$ of ACs have broadly monostratified, multistratified, or diffuse dendrites, and they integrate bipolar cell signals through layer-by-layer summation: ACs with dendrites ramified in multiple strata exhibit $\Delta I_{C} s$ that are sums of $\Delta /_{C} s$ of individual strata. The light-evoked inhibitory chloride current, $\Delta /_{\mathrm{Cl}}$, in strata 1, 2, and 4-6 is ON-OFF (mediated predominantly by ON-OFF ACs or ON ACs plus OFF ACs), and the $\Delta \mathrm{I}_{\mathrm{Cl}}$ in strata 3 and $7-10$ is ON (mediated predominantly by ON ACs). This indicates that the amacrine-amacrine inhibitory synaptic circuitry in the IPL is asymmetrical in favor of the ON channels.

Key words: retina; amacrine cells; bipolar cells; inner plexiform layer; dendritic stratification; ON and OFF visual channels; layer-by-layer signal computation; signal segregation and integration
The visual system encodes and analyzes images of the outside world through parallel channels: different types of neurons are devoted to processing different attributes of visual images, such as brightness, shape, color, contrast, and dynamics, to segregated regions in the brain (Enroth-Cugell and Robson, 1966; Hubel and Wiesel, 1977; Hubel and Livingstone, 1987). The first set of parallel representations of the visual world is embodied in the inner plexiform layer (IPL) of the retina, in which axon terminals of bipolar cells carrying different attributes of visual stimuli stratify at different levels (or strata) (Wu et al., 2000; Roska and Werblin, 2001). It has been established in many vertebrate species that the ON and OFF bipolar cell inputs are segregated in the IPL: synapses of ON cells ramify at the proximal half (sublamina B) and those of the OFF cells ramify at the distal half of the IPL (sublamina A) (Famiglietti and Kolb, 1976; Nelson et al., 1978). In mammalian retinas, one type of rod bipolar cell and 9-10 types of cone bipolar cells with different patterns of axon terminal stratification have been identified (Boycott and Wassle, 1991, 1999; Euler and Wassle, 1995), but the light response characteristics of these cells have not been studied systematically. By comparing light responses and the cell morphology, a recent study has shown that bipolar cells in the salamander retina can be divided into at least 12 types, each of which carries a unique set of light response attributes and bears axons that stratify at different strata of the IPL (Wu et al., 2000). These stacked layers of

Received Nov. 5, 2001; revised Feb. 26, 2002; accepted March 8, 2002.

This work was supported by National Institutes of Health (NIH) Grant EY 04446, NIH Vision Core Grant EY 02520, the Retina Research Foundation (Houston, TX), and Research to Prevent Blindness, Inc. We thank Drs. Bruce Maple and Roy Jacoby for critically reading this manuscript.

Correspondence should be addressed to Dr. Samuel M. Wu, Cullen Eye Institute, Baylor College of Medicine, One Baylor Plaza, NC-205, Houston, TX 77030. E-mail:swu@bcm.tmc.edu.

Copyright (C) 2002 Society for Neuroscience $\quad 0270-6474 / 02 / 224693-09 \$ 15.00 / 0$ axon terminals form a highly organized three-dimensional structure that innervates the third-order retinal cells [amacrine cells (ACs) and ganglion cells] in the IPL. It is not clear, however, how third-order retinal cells process these laminated bipolar cell inputs, and what computational algorithms ACs and ganglion cells use to generate responses from the layer-by-layer representation of bipolar cell signals in the IPL.

ACs are the primary interneurons in the inner retina, and they receive excitatory synaptic inputs from bipolar cells and inhibitory synaptic contacts from other ACs (Wong-Riley, 1974; Marc and Liu, 2000). AC morphology has been studied by using Golgi staining and a fluorescent "photofilling" technique, and they display extreme morphological diversity (Ramon y Cajal, 1893; Vaney, 1990; MacNeil and Masland, 1998; MacNeil et al., 1999). It is not certain, however, how AC morphology is correlated with their light responses and synaptic inputs, and whether ACs with dendrites stratifying in different strata of the IPL carry different sets of light response attributes. Here we report a systematic study on the light response characteristics of 164 morphologically identified ACs in the salamander retina. Light-evoked current responses at various holding potentials were recorded under voltage-clamped conditions so that excitatory and inhibitory synaptic inputs could be separated, and the pattern of dendritic stratification in the IPL of each recorded cell was examined by Lucifer yellow fluorescence with a confocal microscope. Based on the patterns of excitatory and inhibitory light-evoked $\mathrm{ON}$ and OFF responses of the narrowly monostratified ACs, we identify rules and layer-by-layer computational algorithms used by broadly monostratified and multistratified ACs to generate their light responses.

\section{MATERIALS AND METHODS}

Larval tiger salamanders (Ambystoma tigrinum) purchased from Charles D. Sullivan, Co. (Nashville, TN) and KON's Scientific Co. Inc. (Ger- 
mantown, WI) were used in this study. The procedures of dissection, retinal slicing, and recording have been described in previous publications (Werblin, 1978; Wu, 1987). Dissection and recording were done under infrared illumination with a dual-unit Fine-R-Scope (FJW Industry, Mount Prospect, IL) and Nitemare infrared scopes (Meyers, Redmond, WA). Oxygenated Ringer's solution was introduced continuously to the superfusion chamber, and the control Ringer's solution contained (in mM): $108 \mathrm{NaCl}, 2.5 \mathrm{KCl}, 1.2 \mathrm{MgCl}_{2}, 2 \mathrm{CaCl}_{2}$, and $5 \mathrm{HEPES}$, adjusted to a $\mathrm{pH}$ of 7.7. All chemicals were dissolved in control Ringer's solution. A photostimulator was used to deliver light spots $(600-1200 \mu \mathrm{m}$ diameter) to the retina via the epi-illuminator of the microscope. The intensity of unattenuated $(\log \mathrm{I}=0) 500 \mathrm{~nm}$ light was $2.05 \times 10^{7}$ photons $\cdot \mu \mathrm{m}^{-2} \cdot \mathrm{sec}^{-1}$.

Voltage-clamp recordings were made with an Axopatch 200A amplifier connected to a DigiData 1200 interface and pClamp 6.1 software (all from Axon Instruments, Foster City, CA). Patch electrodes of $5 \mathrm{M} \Omega$ tip resistance when filled with internal solution containing (in $\mathrm{mm}$ ): $118 \mathrm{Cs}$ methanesulfonate, $12 \mathrm{CsCl}, 5$ EGTA, $0.5 \mathrm{CaCl}_{2}$, 4 ATP, $0.3 \mathrm{GTP}, 10$ Tris, 0.8 Lucifer yellow, adjusted to a $\mathrm{pH}$ of 7.2 with $\mathrm{CsOH}$, were made with patch electrode pullers from Narishige (Tokyo, Japan) or Sutter Instruments (Novato, CA). The chloride equilibrium potential, $E_{\mathrm{Cl}}$, with this internal solution was approximately $-60 \mathrm{mV}$. Estimates of the liquid junction potential at the tip of the patch electrode before seal formation varied from -9.2 to $-9.6 \mathrm{mV}$. For simplicity, we corrected all holding potentials by $10 \mathrm{mV}$.

Three-dimensional cell morphology was visualized in living retinal slices (250-300 $\mu \mathrm{m}$ in thickness) through the use of Lucifer yellow fluorescence with a confocal microscope (Zeiss 510; Zeiss, Thornwood, NY). Images were acquired with a $40 \times$ water immersion objective (numerical aperture, 0.75 ), using the $458 \mathrm{~nm}$ excitation line of an argon laser and a long-pass $505 \mathrm{~nm}$ emission filter. Consecutive optical sections were superimposed to form a single image using Zeiss Laser Scanning Microscope-PC software, and these compressed image stacks were further processed in Adobe Photoshop 6.0 (Adobe Systems, San Jose, CA) to improve the signal-to-noise ratio. Because signal intensity values were typically enhanced during processing to improve the visibility of smaller processes, the cell bodies and larger processes of some cells appear saturated because of their larger volume of fluorophore. Although the background images of the retinal slices were acquired simultaneously with the fluorescent cells, they were imaged using transmitted light. The level at which dendritic processes stratified in the IPL was characterized by the distance from the processes to the distal margin of the IPL (Fig. $1 A)$. We selected for cells in the AC layer with somas situated beneath the surface of the slice, and they usually had relatively intact processes (assessed by rotation of the stacked images).

\section{RESULTS}

\section{Morphological diversity of amacrine cells}

Figure $1 A$ shows the stacked confocal fluorescent image of an AC (Fig. $1 A, a$ ), a sketch of the same cell on a schematic background of a retina with the IPL divided into 10 strata (Fig. $1 A, b$ ), and the light-evoked current responses to a $0.5 \mathrm{sec}$ light step at various holding potentials (Fig. $1 A, c$ ). Typical AC features include a soma (cell body) centered in the proximal half of the IPL, and dendritic processes ramifying laterally in the IPL. The AC in Figure $1 A$ is an example of what we termed a "broadly monostratified" cell (cells with dendrites ramifying in two to three contiguous strata, in this case in strata 4-6). By using this protocol, we studied a total of 164 ACs. Forty-nine (30\%) were "narrowly monostratified" (with dendrites ramifying in one of the ten strata of the IPL), $32(19 \%)$ were broadly monostratified (dendrites ramifying in two to four contiguous strata), $24(15 \%)$ were "narrowly bistratified" (dendrites ramifying in two noncontiguous strata), $8(5 \%)$ were "broadly bistratified" (dendrites ramified in two bands and at least one band ramifying in two or more contiguous strata), $4(2 \%)$ were "narrowly tristratified" (dendrites ramified in three noncontiguous strata), 11 (7\%) were "broadly tristratified" (dendrites ramified in three bands and at least one band ramifying in two or more contiguous strata), and $36(22 \%)$ were "diffuse" (dendrites ramified in four or more bands or in five or more contiguous strata). The distribution of $\mathrm{AC}$ dendrite stratification levels in the IPL is given in Figure $1 B$. Each vertical column represents an AC and the short vertical bars in individual strata indicate the presence of dendritic processes in that stratum. Cells were arranged in the order of number of their dendritic strata (narrowly monostratified cells first, within which cells were arranged in the order of their dendritic strata 1-10). In this sample of $164 \mathrm{ACs}$, approximately one-half of the ACs are monostratified, three-fifths of which are narrowly ramified in a single stratum. The second largest group is the diffuse ACs (22\%), among which the majority have dendrites distributed contiguously in strata 2-9. There are no clear patterns of dendritic distribution within bistratified and tristratified cells in our data pool.

In addition to the difference in levels of dendritic stratification in the IPL, ACs vary with respect to the width of their dendritic field $\left(W_{\mathrm{D}}\right)$. In our sample of $164 \mathrm{ACs}$, there are $39(24 \%)$ narrow-field cells [N cells; $W_{\mathrm{D}}<125 \mu \mathrm{m}$ (MacNeil and Masland, 1998)], 77 (47\%) medium-field cells (M cells; $125 \mu \mathrm{m}<W_{\mathrm{D}}<400$ $\mu \mathrm{m}$ ), and 48 (29\%) wide-field cells (W cells; $\left.W_{\mathrm{D}}>400 \mu \mathrm{m}\right) \mathrm{s}$. The dendritic field width of each of the 164 cells is labeled in Figure $1 B$ (bottom row). There is no clear correlation between the dendritic field width and the levels of dendritic stratification in the IPL. One exception is that we did not find any wide-field diff use ACs: all wide-field cells were monostratified, bistratified, or tristratified. It is important to note, however, that our voltage-clamp recordings were made from the AC cell bodies, and therefore we might not have space-clamped all dendrites of the ACs, especially those of the wide-field cells. Thus the light-evoked responses of the wide-field cells may be dominated by the synaptic inputs to the dendrites close to the cell bodies. Another potential source of inaccuracy in our measurements is that we recorded ACs from retinal slices. Although we chose to record from ACs several layers under the slice surface (which had more intact dendrites as assessed by image rotation with the confocal microscope; see Materials and Methods), some dendrites may still be cut off during slicing. Therefore, we may have underestimated the AC dendritic field size, especially the $W_{\mathrm{D}}$ of the wide-field cells.

\section{Light-evoked current responses of morphologically identified amacrine cells}

Figure 2 shows stacked confocal fluorescent images (Fig. 2A,C) and light-evoked current responses at various holding potentials (Fig. 2B,D) of eight ACs. Cells $a-d$ are narrowly monostratified at strata $1,3,7$, and 9 , respectively; cell $e$ is broadly tristratified at strata $3,6,7$, and 9; cell $f$ is narrowly bistratified at strata 3 and 8 ; cell $g$ is broadly monostratified at strata 9 and 10; and cell $h$ is diffuse across strata $1-9$. Cells $b, e$, and $f$ are narrow-field cells; cells $a, c$, and $h$ are medium-field cells; and cells $d$ and $g$ are wide-field cells. To a $0.5 \mathrm{sec}$ light step measured at $-60 \mathrm{mV}$, the excitatory light-evoked cation current response $\left(\Delta I_{\mathrm{C}}\right)$ of cell $a$ was a transient inward current at light offset (OFF response), the $\Delta I_{\mathrm{C}} \mathrm{s}$ of cells $b-d, f$, and $g$ were transient inward currents at light onset (ON response), and the $\Delta I_{\mathrm{C}} \mathrm{s}$ of cells $e$ and $h$ were transient inward currents at both light onset and offset (ON-OFF response). The inhibitory light-evoked chloride current responses $\left(\Delta I_{\mathrm{Cl}} \mathrm{s}\right)$ measured at $0 \mathrm{mV}$ of cells $a$ and $f-h$ were ON-OFF transient outward currents, and those in cells $b-e$ were ON transient outward currents. The current responses measured at $+40 \mathrm{mV}$ and $-100 \mathrm{mV}$ in each cell were mediated by the light-evoked cation and chloride conductance changes $\left(\Delta g_{\mathrm{C}}\right.$ and $\Delta g_{\mathrm{Cl}}$, respectively). 
A a

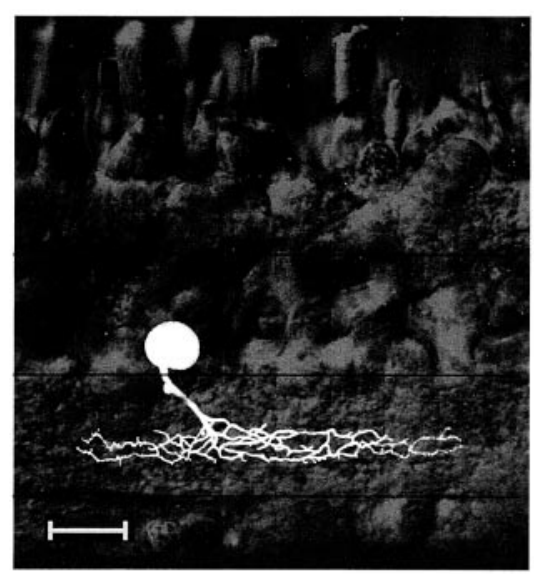

b

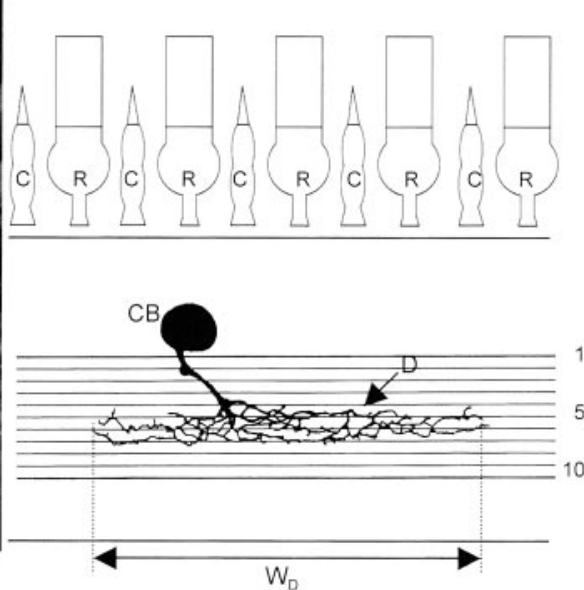

c

PRL

OPL

INL

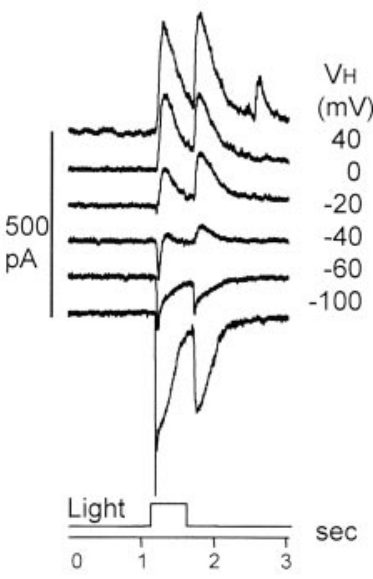

B

IPL

Narrowly Monostratified, $n=49$

Broadly Monostratified, $n=32$

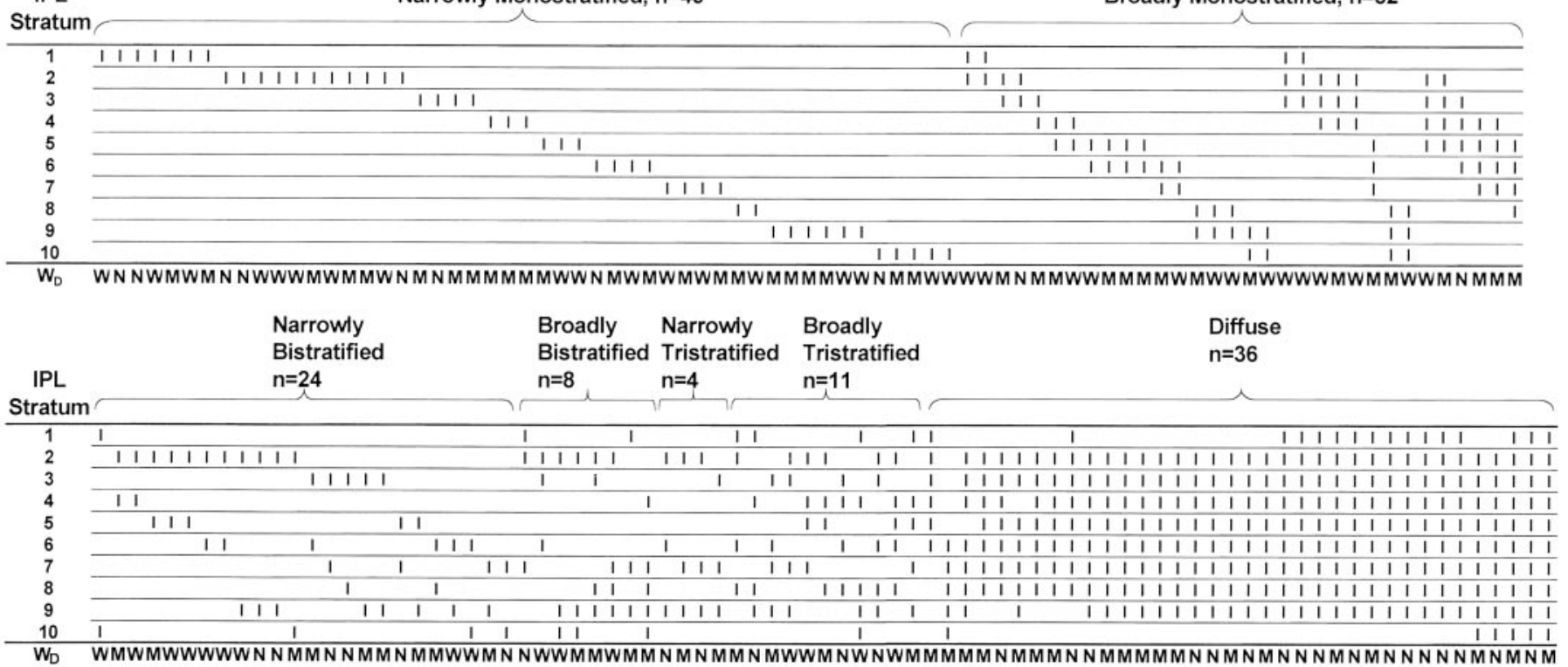

Figure 1. A, A stacked confocal fluorescent image of an $\mathrm{AC}(a)$, a sketch of the same cell on a schematic background of the retina with marked divisions of 10 strata of the IPL $(b)$, and the light-evoked current responses to a $0.5 \mathrm{sec}$ light step [500 $\mathrm{nm},-3.3$ (3.3 log unit attenuation)] at various holding potentials $(c)$. $C B$, Cell body; $D$, dendrites; $R$, rod; $C$, cone; $P R L$, photoreceptor layer; $O P L$, outer plexiform layer; $I N L$, inner nuclear layer; $G C L$, ganglion cell layer. Scale bar, $25 \mu \mathrm{m}$. B, Distribution of dendritic stratification levels in the IPL of 164 ACs. These cells are grouped as narrowly monostratified, broadly monostratified, narrowly bistratified, broadly bistratified, narrowly tristratified, broadly tristratified, and diff use cells (definitions are given in Results). Each vertical column represents an AC, and the short vertical bars in individual strata indicate the presence of dendritic processes in that stratum. The $W_{\mathrm{D}}$ of each of the 164 cells is given at the bottom of each panel in $B$, as follows: $N$, Narrow field; $M$, medium field; $W$, wide field (definitions are given in Results).

\section{Layer-by-layer analysis of excitatory and inhibitory light-evoked ON and OFF signals in narrowly monostratified amacrine cells}

To determine the stratum-by-stratum excitatory and inhibitory synaptic inputs, we analyzed the $\Delta I_{\mathrm{C}}$ and $\Delta I_{\mathrm{Cl}}$ of narrowly monostratified ACs, because these cells make synaptic contacts with bipolar cells and other ACs in one IPL stratum. Figure 3 shows the $\Delta I_{\mathrm{C}}$ and $\Delta I_{\mathrm{C} 1}$ (Fig. 3, bottom panel) of 10 (marked as cells $1-10$, corresponding to the stratum each cell ramifies) narrowly monostratified cells whose dendrites ramified in each of the 10 strata of the IPL. The top panel of Figure 3 shows sketches (based on stacked confocal fluorescent images; same procedure as shown in Fig. $1 A$ ) of the AC bodies and a portion of the narrowly monostratified dendrites near the soma (extended portions of dendrites of medium- and wide-field cells were cut off to save space) on a schematic background of the retina. The dendritic thickness (in the dimension parallel with the photoreceptor axes) of the majority $(75-80 \%)$ of the narrowly monostratified cells is approximately one-tenth the thickness of the IPL, whereas $\sim 10 \%$ are slightly wider than one-tenth of the IPL and the rest are somewhat thinner than one-tenth of the IPL. At $-60 \mathrm{mV}$, cells 1 , 2 , and 4 exhibited an OFF $\Delta I_{C}$; cells 3 and $7-10$ gave rise to an ON $\Delta I_{\mathrm{C}}$; and cells 5 and 6 gave an ON-OFF $\Delta I_{\mathrm{C}}$. This pattern of excitatory light responses was consistent in all narrowly monostratified ACs within a given stratum, regardless of their dendritic field size (for example, cells $a-d$ in Fig. 2). The average $\pm \mathrm{SD}$ peak amplitudes of $\Delta I_{\mathrm{C}}$ in various types of narrowly monostratified ACs are given in Table 1, and they suggest that the excitatory 

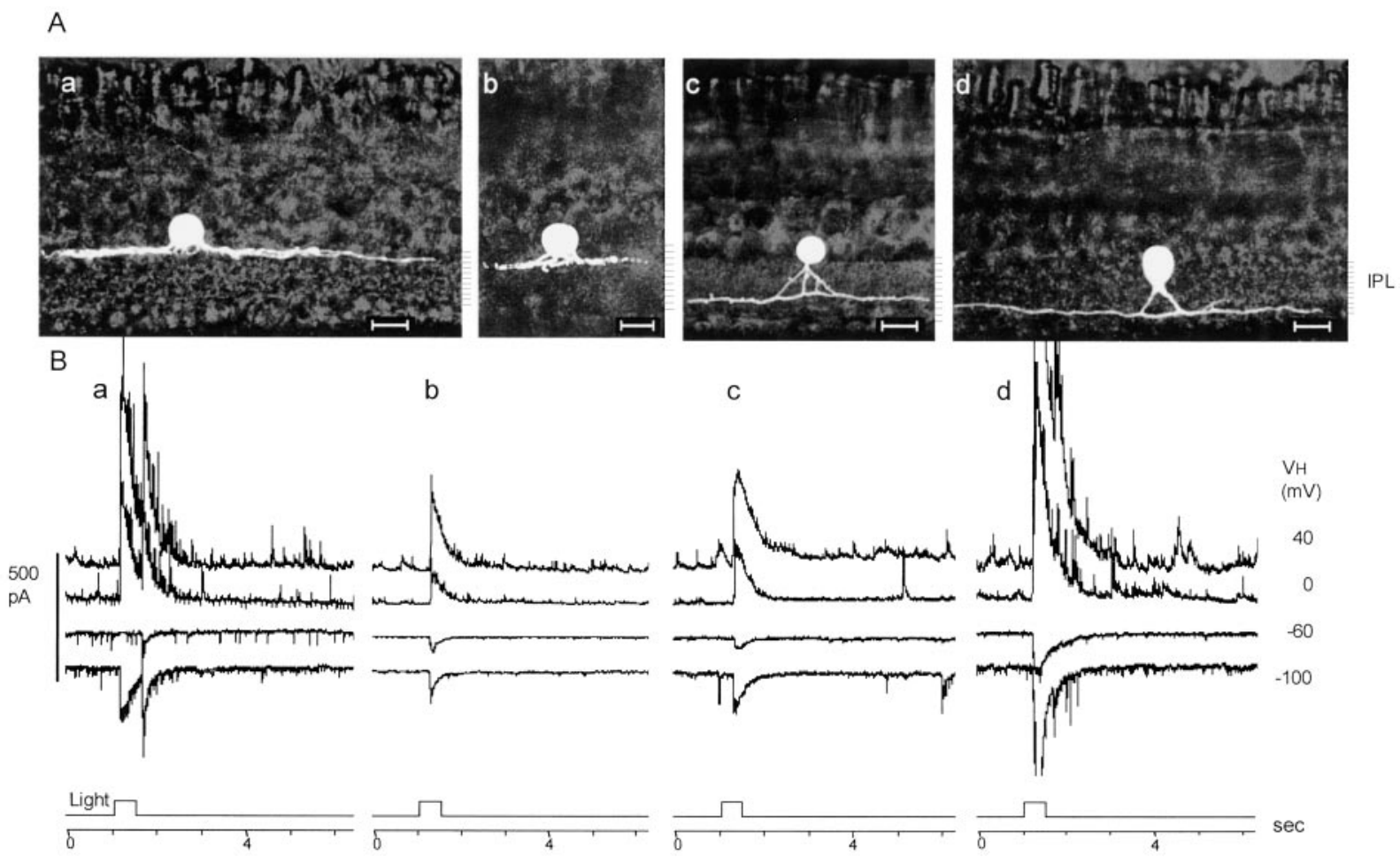

C
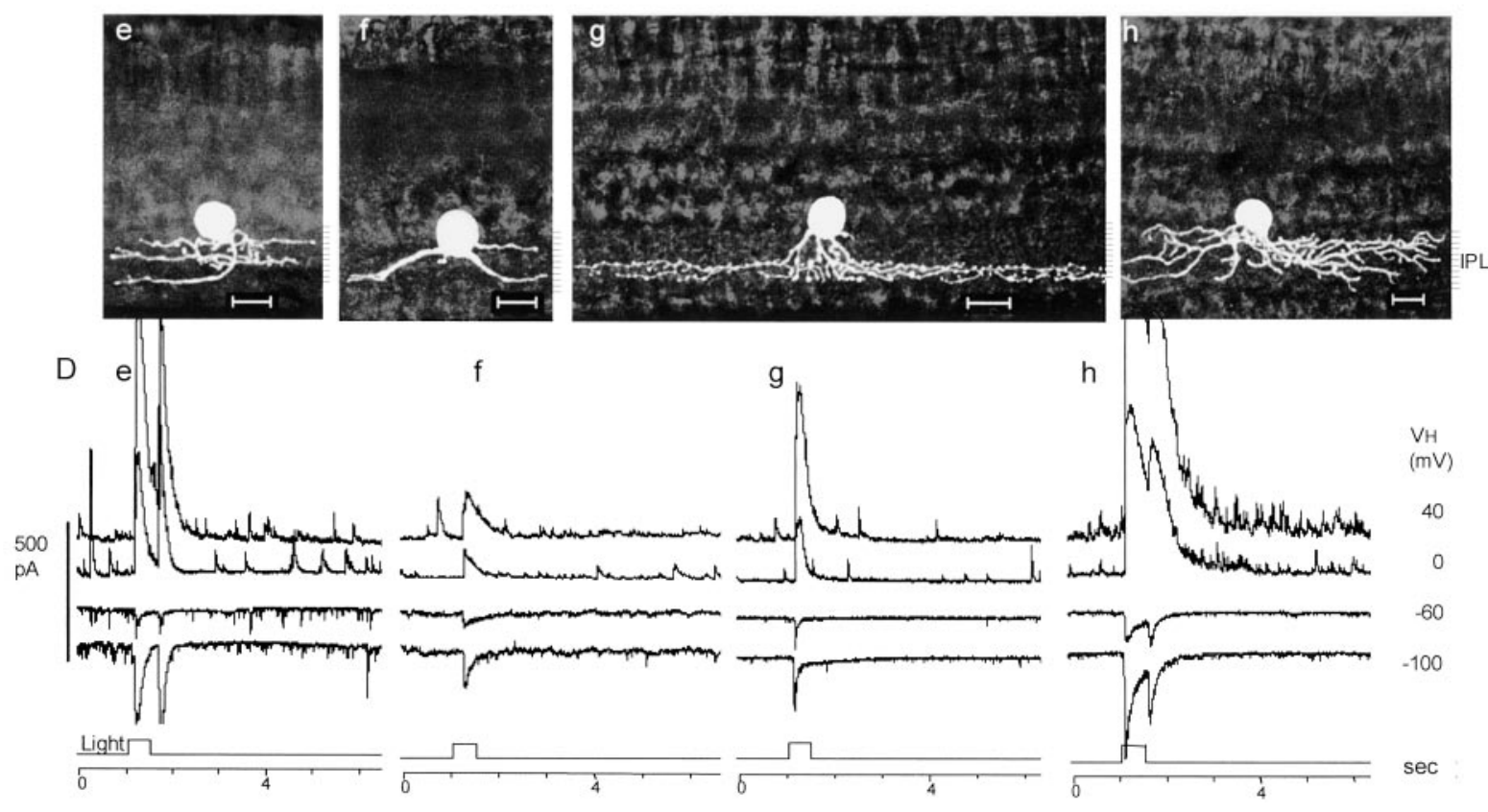

Figure 2. Stacked confocal fluorescent images $(A, C)$ and current responses evoked by a $0.5 \mathrm{sec}$ light step $(500 \mathrm{~nm},-3.3)$ at various holding potentials $(40,0,-60$, and $-100 \mathrm{mV})(B, D)$ of eight $(a-h)$ ACs. The ten strata of the IPL in each image are marked by scales on the right. Scale bars, $25 \mu \mathrm{m}$. The excitatory light-evoked cation current response $\left(\Delta I_{\mathrm{C}}\right)$ of each cell was measured at $E_{\mathrm{Cl}}(-60 \mathrm{mV})$, and the inhibitory light-evoked chloride current response $\left(\Delta I_{\mathrm{Cl}}\right)$ was measured at $E_{\mathrm{C}}(0 \mathrm{mV})$.

inputs to strata 1,2 , and 4 are predominantly mediated by hyperpolarizing bipolar cells (HBCs); those to strata 3 and 7-10 are predominantly mediated by the depolarizing bipolar cells (DBCs); and those to strata 5 and 6 are mediated by both HBCs and DBCs.

At $0 \mathrm{mV}$, cells 1,2 , and $4-6$ showed $\mathrm{ON}-\mathrm{OFF} \Delta I_{\mathrm{Cl}} \mathrm{s}$ and cells
3 and 7-10 exhibited $\mathrm{ON} \Delta I_{\mathrm{Cl}}$ s, indicating that ACs making inhibitory synaptic contacts with cells 1,2 , and 4-6 (those with OFF $\Delta I_{C} s$ ) gave rise to ON-OFF light responses, and ACs making inhibitory synaptic contacts with cells 3 and 7-10 (those with only $\mathrm{ON} \Delta I_{\mathrm{C}} \mathrm{s}$ ) gave $\mathrm{ON}$ light responses. This pattern of inhibitory light responses is also consistent in all narrowly mono- 


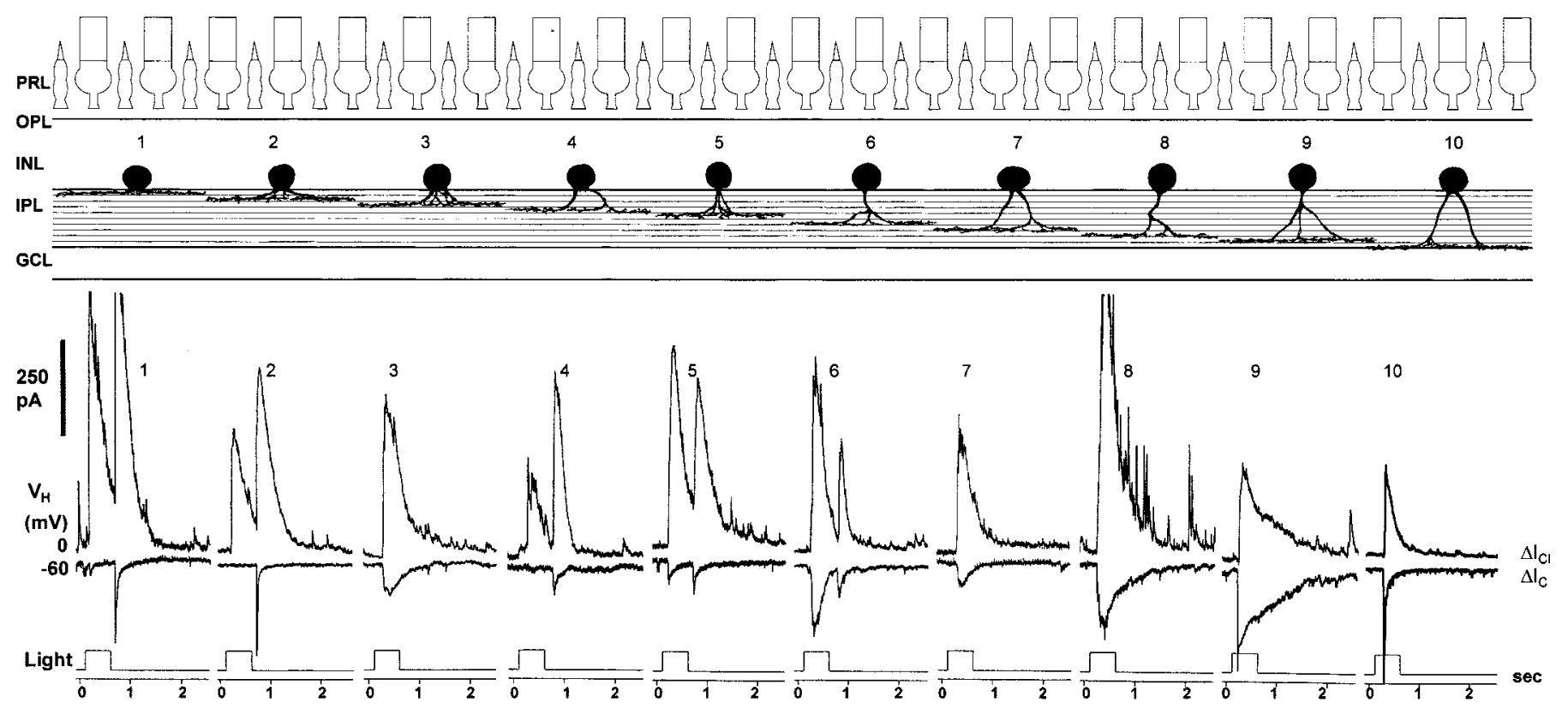

Figure 3. Top, Sketches based on stacked confocal fluorescent images (same procedure as shown in Fig. 1A) of 10 (cells 1-10, corresponding to the stratum each cell ramifies) narrowly monostratified ACs. Extended portions of dendrites of medium- and wide-field cells were cut off to save space. $P R L$, Photoreceptor layer; $O P L$, outer plexiform layer; $I N L$, inner nuclear layer; $G C L$, ganglion cell layer. Bottom, Current responses evoked by a 0.5 sec light step $(500 \mathrm{~nm},-3.3)$ at holding potentials of $-60 \mathrm{mV}\left(\Delta I_{\mathrm{C}}\right)$ and $0 \mathrm{mV}\left(\Delta I_{\mathrm{Cl}}\right)$.

Table 1. The average \pm SD peak amplitudes, time-to-peak of the rising phase $\left(\tau_{0}\right)$, and decay time constant $\left(\tau_{\mathrm{D}}\right.$, fitted by single exponentials) of $\Delta I_{\mathrm{C}}$ and $\Delta I_{\mathrm{Cl}}$ in various types of narrowly monostratified $\mathrm{ACs}$

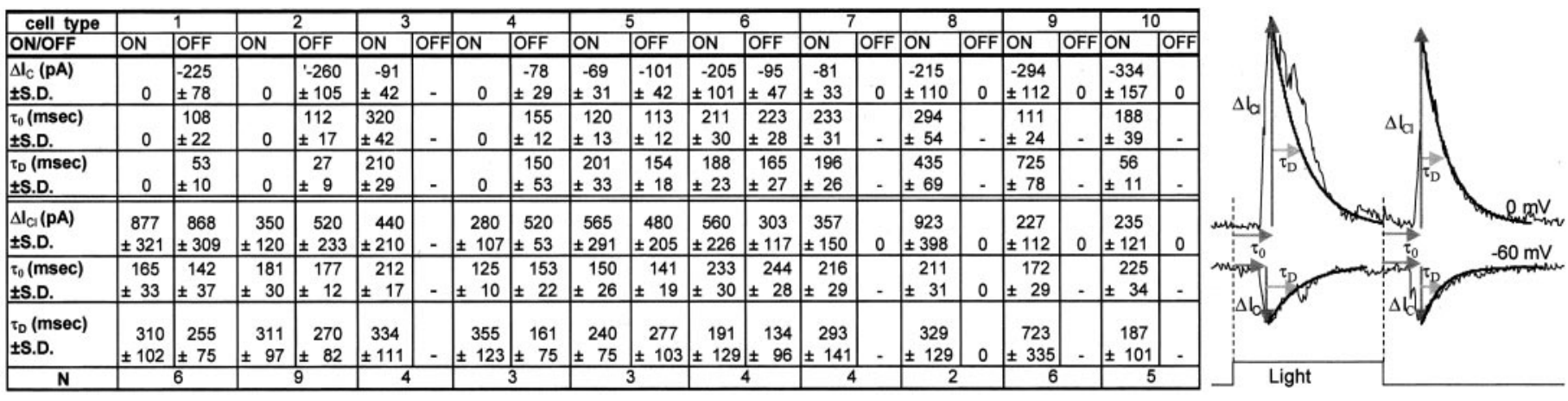

The light step in the inset is $0.5 \mathrm{sec}$ long $(500 \mathrm{~nm},-3.3)$, and $\Delta I_{\mathrm{C}}$ and $\Delta I_{\mathrm{Cl}}$ were measured at the onset (ON) and offset (OFF) of the light step.

stratified ACs within a given stratum, regardless of their dendritic field size (for example, cells $a-d$ in Fig. 2). The average $\pm \mathrm{SD}$ peak amplitudes of $\Delta I_{\mathrm{Cl}}$ of various types of narrowly monostratified ACs are also listed in Table 1, and they suggest that the inhibitory inputs to strata 1,2 , and 4 (strata with OFF $\Delta I_{C} s$ ) and strata 5 and 6 (strata with $\mathrm{ON}-\mathrm{OFF} \Delta I_{\mathrm{C}} \mathrm{s}$ ) are mediated predominantly by $\mathrm{ON}-\mathrm{OFF} \mathrm{ACs}$, and that those to strata 3 and 7-10 (strata with $\mathrm{ON} \Delta I_{C} \mathrm{~s}$ ) are mediated predominantly by ON ACs.

In addition to differences in $\mathrm{ON}$ and OFF inputs, the kinetics of $\Delta I_{\mathrm{C}}$ and $\Delta I_{\mathrm{Cl}}$ in various types of narrowly monostratified ACs was different, and the average $\pm \mathrm{SD}$ rise time $\left(\tau_{0}\right)$ and decay time constant $\left(\tau_{\mathrm{D}}\right)$ of $\Delta I_{\mathrm{C}}$ and $\Delta I_{\mathrm{C} 1}$ are listed in Table 1 . The kinetics of cells 1, 2, and 10 was faster than that of the rest. The mean decay time constant $\left(\tau_{\mathrm{D}}\right)$ of cells 1,2 , and 10 , for example, ranged from 27 to $56 \mathrm{msec}$, whereas that of cells 3-9 varied from 150 to 725 msec. Because the axon terminals of rod-dominated HBCs ramify primarily in strata 1 and 2, and axon terminals of roddominated DBCs ramify primarily in stratum 10 (Wu et al.,
2000), this result suggests that the postsynaptic responses of rod-dominated bipolar cells in ACs are more transient than the postsynaptic responses of cone-dominated bipolar cells. It is not clear why rod-dominated inputs are more transient in ACs. Because the light responses of rod-dominated bipolar cells are not more transient than the cone-dominated bipolar cell responses (Yang and Wu, 1997; Wu et al., 2000), the difference in AC response kinetics is probably mediated by differences in the amacrine-amacrine and amacrine-bipolar synaptic network. Additional studies are needed to clarify this issue.

\section{Layer-by-layer computation of ON and OFF signals in broadly monostratified and multistratified amacrine cells}

We subsequently examined whether the rules segregating $\mathrm{ON}$ and OFF channels in various IPL strata set forth by the narrowly monostratified cells are followed by broadly monostratified and multistratified (bistratified, tristratified, and diffuse) ACs. Figure 


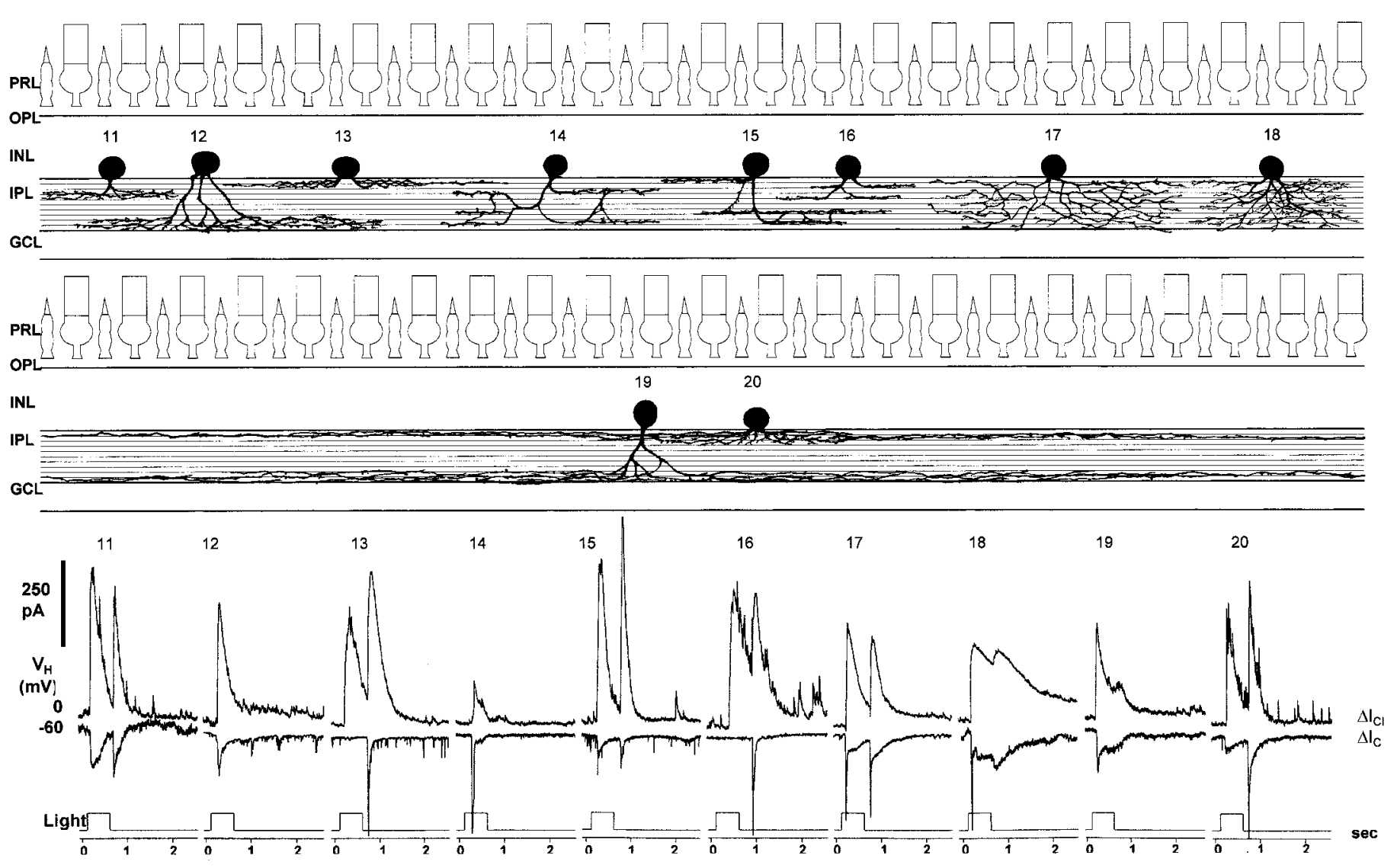

Figure 4. Top, Sketches based on stacked confocal fluorescent images of 10 (cells 11-20) broadly monostratified (cells 11-13, 19, and 20), narrowly bistratified (cell 16), narrowly tristratified (cells 14 and 15), and diff use (cells 17 and 18) ACs. PRL, Photoreceptor layer; OPL, outer plexiform layer; $I N L$, inner nuclear layer; $G C L$, ganglion cell layer. Bottom, Current responses evoked by a $0.5 \mathrm{sec}$ light step $(500 \mathrm{~nm},-3.3)$ at holding potentials of -60 $\mathrm{mV}\left(\Delta I_{\mathrm{C}}\right)$ and $0 \mathrm{mV}\left(\Delta I_{\mathrm{Cl}}\right)$.

4 shows the morphology (top panel) and $\Delta I_{\mathrm{C} 1}$ and $\Delta I_{\mathrm{C}}$ (bottom panel) of 10 (cells 11-20) ACs. Cell 11 was broadly monostratified in strata 3 (ON stratum) and 4 (OFF stratum), and $\Delta I_{C}$ s and $\Delta I_{\mathrm{Cl}}$ s were $\mathrm{ON}-\mathrm{OFF}$. Cell 12 was broadly monostratified in strata 8 (ON stratum), 9 (ON stratum), and 10 (ON stratum), and $\Delta I_{\mathrm{C}} \mathrm{s}$ and $\Delta I_{\mathrm{Cl}} \mathrm{s}$ were $\mathrm{ON}$. Cell 13 was broadly monostratified in strata 1 (OFF stratum) and 2 (OFF stratum), the $\Delta I_{\mathrm{C}}$ was OFF, and the $\Delta I_{\mathrm{Cl}}$ was $\mathrm{ON}-\mathrm{OFF}$, whereas cell 14 was narrowly tristratified in strata 3 (ON stratum), 7 (ON stratum), and 9 (ON stratum), and $\Delta I_{\mathrm{C}} \mathrm{s}$ and $\Delta I_{\mathrm{Cl}} \mathrm{s}$ were $\mathrm{ON}$. Cell 15 was narrowly tristratified in strata 1 (OFF stratum), 7 (ON stratum), and 9 (ON stratum), and $\Delta I_{\mathrm{C}} \mathrm{s}$ and $\Delta I_{\mathrm{Cl}} \mathrm{s}$ were $\mathrm{ON}-\mathrm{OFF}$, and cell 16 was narrowly bistratified in strata 2 (OFF stratum) and 4 (OFF stratum), the $\Delta I_{\mathrm{C}}$ was OFF, and the $\Delta I_{\mathrm{Cl}}$ was $\mathrm{ON}$. Cells 17 and 18 were diff use across all strata (ON and OFF strata), and $\Delta I_{\mathrm{C}}$ s and $\Delta I_{\mathrm{Cl}}$ s were ON-OFF. Cell 19 (wide dendritic field) was broadly monostratified in strata 9 (ON stratum) and 10 (ON stratum), and $\Delta I_{\mathrm{C}} \mathrm{S}$ and $\Delta I_{\mathrm{Cl}} \mathrm{S}$ were $\mathrm{ON}$, and cell 20 was broadly monostratified in strata 1 (OFF stratum), 2 (OFF stratum), and 3 (ON stratum), and $\Delta I_{\mathrm{C}} \mathrm{s}$ and $\Delta I_{\mathrm{Cl}} \mathrm{s}$ were $\mathrm{ON}-\mathrm{OFF}$.

In addition, as shown in Figure 2, cell $e$ was broadly tristratified in strata 3 (ON stratum), 6 (ON-OFF stratum), 7 (OFF stratum), and 10 (ON stratum), and $\Delta I_{\mathrm{C}} \mathrm{s}$ and $\Delta I_{\mathrm{Cl}} \mathrm{s}$ were ON-OFF. Cell $f$ was narrowly bistratified in strata 3 (ON stratum) and 8 (ON stratum), and $\Delta I_{\mathrm{C}} \mathrm{s}$ and $\Delta I_{\mathrm{Cl}} \mathrm{s}$ were $\mathrm{ON}-\mathrm{OFF}$. Cell $g$ was broadly monostratified in strata 9 (ON stratum) and 10 (ON stratum), and $\Delta I_{\mathrm{C}} \mathrm{S}$ and $\Delta I_{\mathrm{Cl}} \mathrm{s}$ were $\mathrm{ON}$, and cell $h$ ramified diffusely across strata
1-9 (ON and OFF strata), and $\Delta I_{\mathrm{C}} \mathrm{s}$ and $\Delta I_{\mathrm{Cl}} \mathrm{s}$ were $\mathrm{ON}-\mathrm{OFF}$. We also examined the correlation between $\mathrm{ON}-\mathrm{OFF} \Delta I_{\mathrm{C}}$ and $\Delta I_{\mathrm{Cl}}$ responses and patterns of dendritic stratification of the remaining 101 multistratified ACs listed in Figure $1 B$. All but five diffuse ACs (which showed ON instead of ON-OFF $\Delta I_{\mathrm{C}}$ and $\Delta I_{\mathrm{Cl}}$ ) followed the ON-OFF rules for the $\Delta I_{\mathrm{C}}$ and $\Delta I_{\mathrm{Cl}}$ responses: cells with dendrites in strata 1, 2, and 4 exhibited an OFF $\Delta I_{\mathrm{C}}$ and an ON-OFF $\Delta I_{\mathrm{Cl}}$; cells with dendrites in strata 3 and 7-10 gave rise to an $\mathrm{ON} \Delta I_{\mathrm{C}}$ and $\Delta I_{\mathrm{Cl}}$; and cells with dendrites in strata 5 and 6 or with diffuse dendrites gave rise to an $\mathrm{ON}-\mathrm{OFF}$ $\Delta I_{\mathrm{C}}$ and $\Delta I_{\mathrm{Cl}}$.

It is clear from these results that the rules correlating $\mathrm{ON}-\mathrm{OFF}$ channels and dendritic stratification in the IPL set forth by narrowly monostratified cells (Fig. 3 and Table 1) are closely followed by all 79 broadly monostratified, bistratified, and tristratified ACs, and by the vast majority (31 of 36) of diff use ACs. Although the amplitudes of the broadly monostratified cells and the responses of the multistratified ACs did not necessarily correlate with the amplitudes of narrowly monostratified $\mathrm{AC}$ responses, the kinetics of $\Delta I_{\mathrm{C}}$ and $\Delta I_{\mathrm{Cl}}$ was very similar to that of the narrowly monostratified ACs ramifying in the strata in which the broadly monostratified and multistratified cells have dendritic branches. These results suggest that ACs with dendrites in multiple strata compute their signals in a layer-by-layer (stratum-bystratum) manner. At least for the ON-OFF signals, responses of broadly monostratified and multistratified cells seem to result 


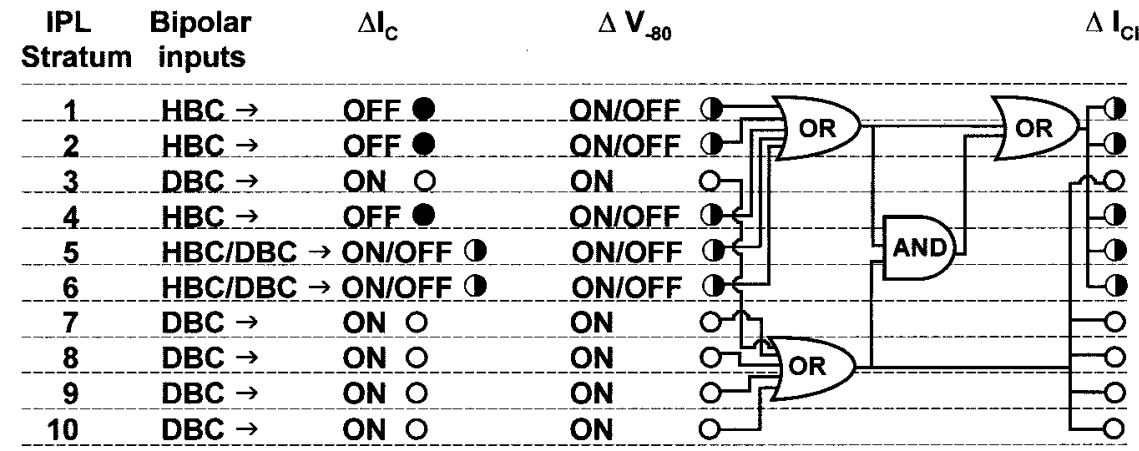

Figure 5. Logic circuit diagram of amacrine-amacrine synaptic connections in the IPL. $\Delta I_{\mathrm{C}}, \Delta V_{-80}$, and $\Delta I_{\mathrm{Cl}}$ are light-evoked excitatory current, lightevoked voltage response at $-80 \mathrm{mV}$, and light-evoked chloride current, respectively. Open circles are ON responses, filled circles are OFF responses, and halfopen/half-filled circles are $\mathrm{ON}-\mathrm{OFF}$ responses. $=$ an and and logic OR and AND gates respectively. from summing up responses of the individual dendritic strata of the cells.

\section{Amacrine-amacrine synaptic circuitry in the IPL}

In the salamander retina, the dark membrane potential of ACs is near $-80 \mathrm{mV}$ (Yang et al., 2002), and $E_{\mathrm{Cl}}$ values in amphibian $\mathrm{ACs}$ are in the range of -50 to $-70 \mathrm{mV}$ (Miller and Dacheux, 1983). Therefore the light-evoked voltage responses are mediated by both $\Delta I_{\mathrm{C}}$ and $\Delta I_{\mathrm{Cl}}$, and thus the ON-OFF pattern of voltage responses at $-80 \mathrm{mV}(\Delta V)$ of each $\mathrm{AC}$ is determined by the ON-OFF pattern of $\Delta I_{\mathrm{C}}$ and $\Delta I_{\mathrm{C} 1}$ (the $I-V$ relationship of the light-evoked current is approximately linear between $0 \mathrm{mV}$ and $-100 \mathrm{mV}$ ) (Yang et al., 2002). Consequently, if all ACs have dark membrane potentials near $-80 \mathrm{mV}$, then narrowly monostratified cell types 1, 2, and 4-6 may receive inhibitory inputs from themselves, from other $\mathrm{ACs}$ with an $\mathrm{ON}-\mathrm{OFF} \Delta V$, or from at least one type of $\mathrm{AC}$ with an $\mathrm{ON}-\mathrm{OFF} \Delta V$ and some ACs with an $\mathrm{ON} \Delta V$. However, narrowly monostratified cell types 3 and 7-10 can receive inhibitory inputs only from themselves or from other ACs with an ON $\Delta V$. These amacrine-amacrine synaptic connections in the 10 IPL strata are summarized by a logic circuit diagram (Tokheim, 1994) in Figure 5. This logic circuit gives all possible connections made by ACs on narrowly monostratified ACs in each stratum. The number of possible connections is very large, because for each OR (any or all) gate of five inputs, there are

$$
\sum_{k=1}^{5} C_{k}^{5}
$$

31 possible connections (where $C_{k}^{5}$ is the number of combinations with $k$ out of five strata), and for the AND (all or nothing) gate with two inputs from the OR gates, there are $31 \times 31=961$ possible connections. Therefore there are $31+961=992$ possible connections that mediate amacrine cell inputs $\left(\Delta I_{\mathrm{Cl}}\right)$ to the narrowly monostratified ACs in strata 1,2 , and 4-6 and 31 possible connections that mediate amacrine cell inputs $\left(\Delta I_{\mathrm{Cl}}\right)$ to the narrowly monostratified ACs in strata 3 and 7-10. Figure 5 clearly indicates that the amacrine-amacrine synaptic circuitry is asymmetric in favor of the ON channels: ACs with ON responses may make synaptic connections with ACs in all 10 strata, whereas ACs with ON and OFF responses may make synaptic connections only with ACs in strata 1,2, and 4-6.

The logic circuit in Figure 5 is based on the finding that most ACs in dark-adapted salamander retina have a dark membrane potential near $-80 \mathrm{mV}$ (Yang et al., 2002). However, for those ACs with dark membrane potentials near $-60 \mathrm{mV}$ or above (Vallerga, 1981), the ON-OFF patterns of the voltage responses of the cell $(\Delta V)$ are different from the $\Delta V_{-80}$ listed in Figure 5, and more complex logic circuits are needed. In addition, analysis of cells $e-h$ in Figure 2 and cells 11-20 in Figure 4 shows that the layer-by-layer rule for the ON-OFF responses applies for amacrine-amacrine inputs $\left(\Delta I_{\mathrm{Cl}}\right)$ in broadly monostratified and multistratified ACs; therefore $\Delta I_{\mathrm{Cl}} \mathrm{s}$ of ACs with dendrites ramified in multiple strata may be computed as sums of $\Delta I_{\mathrm{Cl}} \mathrm{S}$ of individual strata.

\section{DISCUSSION}

Bipolar cell inputs to amacrine cells are organized in a layer-by-layer manner in the IPL: segregation and integration of ON and OFF signals

A major finding of this study is that the light-evoked excitatory synaptic inputs from $\mathrm{ON}$ and $\mathrm{OFF}$ bipolar cells to $\mathrm{ACs}, \Delta I_{\mathrm{C}}$, are organized in a layer-by-layer manner with 10 strata in the IPL. This functional architecture resembles many parallel information pathways in higher visual centers, such as the columnar segregation of neurons registering different orientations in the visual cortex (Hubel and Wiesel, 1968, 1969). Our data show that $~ 30 \%$ of ACs are narrowly monostratified (Fig. $1 A$ ), and they ramify in each of the 10 strata of the IPL (types 1-10 for strata 1-10). Responses of these cells demonstrate that the $\mathrm{ON}$ and $\mathrm{OFF}$ channels are segregated in 10 strata in the IPL: $\Delta I_{\mathrm{C}} \mathrm{S}$ in strata 1 , 2 , and 4 are OFF responses (predominantly mediated by the OFF bipolar cells); $\Delta I_{\mathrm{C}} \mathrm{s}$ in strata 3 and $7-10$ are $\mathrm{ON}$ responses (predominantly mediated by $\mathrm{ON}$ bipolar cells); and $\Delta I_{\mathrm{C}} \mathrm{s}$ in strata 5 and 6 are $\mathrm{ON}-\mathrm{OFF}$ responses (mediated by both $\mathrm{ON}$ and $\mathrm{OFF}$ bipolar cells). Among the 12 types of bipolar cells in the tiger salamander retina, only five types have monostratified axon terminals, whereas the axons of others are bistratified, tristratified, or pyramidally branching (Wu et al., 2000). Therefore, some strata in the IPL may contain axon terminals primarily from one type of bipolar cell, whereas other strata may contain axon terminals from several types of bipolar cells. What our data suggest, nevertheless, is that the inputs from bipolar cells to ACs in each stratum are the same within that stratum: ON, OFF, or $\mathrm{ON}-\mathrm{OFF}$.

Our data suggest that narrowly monostratified ACs carry segregated bipolar cell signals in the inner retina. In contrast, broadly monostratified and multistratified cells integrate bipolar cell signals through layer-by-layer summation: ACs with dendrites ramified in multiple strata exhibit $\Delta I_{\mathrm{C}}$ that are sums of $\Delta I_{\mathrm{C}} \mathrm{s}$ of individual strata (Fig. 4). This suggests that the ON and OFF bipolar cell inputs to ACs (monostratified, multistratified, narrowly stratified, or broadly stratified) in each stratum are very similar, and that $\Delta I_{\mathrm{C}} \mathrm{s}$ from each stratum are transmitted to the soma in a parallel (and thus additive) manner. This is consistent with previous studies that have shown that most bipolar cells, 
either ON (DBCs) or OFF (HBCs), have synaptic terminals ramifying in a restricted stratum (or strata) of the IPL (Wu et al., 2000). Although it is difficult to match all bipolar cell lightresponse attributes with $\Delta I_{\mathrm{C}} \mathrm{s}$ in $\mathrm{ACs}$, our results demonstrate a clear stratum-by-stratum rule for the $\mathrm{ON}-\mathrm{OFF}$ response channels (set forth in Fig. 3 and Table 1) in the IPL. This rule primarily agrees with the sublamina A/B rule for the OFF-ON cells established by previous studies in the cat retina (Famiglietti and Kolb, 1976; Nelson et al., 1978) and salamander retina (Pang et al., 2002). An obvious exception is that we found that the $\Delta I_{\mathrm{C}}$ in stratum 3 (located in the middle of sublamina A) exhibits $\mathrm{ON}$ instead of OFF responses. Previous studies in salamanders and turtles have shown that there are DBCs that bear axon terminals ramifying in two strata in the IPL, one in sublamina A and the other in sublamina B (Ammermuller and Kolb, 1995; Wu et al., 2000). Recent results have shown that antibodies against G-protein $\alpha$-subunit stain DBCs in the salamander retina, and in addition to heavy labeling in sublamina B, there is a clear band near stratum 3 in sublamina A (J. Zhang and S. M. Wu, unpublished results). It is possible, at least in the tiger salamander retina, that the $\mathrm{ON}$ channels mediated by $\mathrm{DBC}$ s activate synapses not only in sublamina B but also in the middle of sublamina A (stratum 3). This may partially explain why the vast majority of ACs and ganglion cells in the salamander retina are ON-OFF or ON cells, and only $\sim 5-10 \%$ of these third-order cells are OFF cells (Hensley et al., 1993; Gao and Wu, 1998; Yang et al., 2002).

At first glance, the division of the IPL into 10 strata appears arbitrary. However, the radial dendritic thickness of the majority of the narrowly stratified cells (either monostratified or multistratified) is very close to one-tenth the thickness of the IPL (only $\sim 10 \%$ are slightly wider than one-tenth of the IPL, and $\sim 10-$ $15 \%$ are thinner than one-tenth of the IPL). This provides the anatomical rationale for the 10 strata scheme. In addition, our physiological data also support this scheme. For example, cells 2 and 4 in Figure 3 (narrowly monostratified in strata 2 and 4 , respectively) are OFF cells, but cell 3 , which has dendrites ramified approximately one-tenth of the IPL thickness away from the dendrites of cells 2 and 4, is an ON cell. Table 1 and Figure 4 show that this clear physiological difference can be observed in all monostratified and multistratified cells that bear narrowly stratified dendrites in strata 2-4. Another example is that although cells 9 and 10 (Fig. 3 ) are both ON cells, their $\Delta I_{C}$ kinetics is quite different. Table 1 shows that the $\Delta I_{\mathrm{C}}$ decay time constant $\left(\tau_{\mathrm{D}}\right)$ of cells in stratum 9 is $725 \pm 78$, and that the decay time constant of cells in stratum 10 is $56 \pm 11$. Therefore, the morphological and physiological data we obtained support the 10 strata scheme for classifying ACs in the tiger salamander retina.

\section{Amacrine-amacrine synaptic interactions in the IPL: asymmetric synaptic circuitry in favor of the ON channels}

Our data show that the inhibitory synaptic inputs to ACs are more complex than the excitatory inputs. Responses of narrowly monostratified ACs suggest that the light-evoked inhibitory inputs, $\Delta I_{\mathrm{Cl}}$, to strata 1,2 , and 4 (strata with OFF $\Delta I_{\mathrm{C}} \mathrm{s}$ ) and strata 5 and 6 (strata with $\mathrm{ON}-\mathrm{OFF} \Delta I_{\mathrm{C}} \mathrm{s}$ ) are $\mathrm{ON}-\mathrm{OFF}$ (mediated predominantly by $\mathrm{ON}-\mathrm{OFF} \mathrm{ACs}$ ), and that those to strata 3 and 7-10 (strata with ON $\Delta I_{\mathrm{C}}$ s from DBCs) are ON (mediated predominantly by ON ACs). This indicates that the narrowly monostratified cells receiving excitatory inputs from OFF bipolar cells (types 1,2, and 4) must receive inhibitory inputs from $O N$ and OFF channels mediated by both $\mathrm{ON}$ and OFF bipolar cells.
However, narrowly monostratified cells receiving excitatory inputs from ON bipolar cells (types 3 and 7-10) receive inhibitory inputs only from the ON channel mediated by the ON bipolar cells. Therefore, at least in the tiger salamander, the amacrineamacrine synaptic circuitry in the IPL seems asymmetric in favor of the ON channels. The logic circuit diagram in Figure 5 clearly demonstrates this asymmetry: ACs with $\mathrm{ON}$ responses may make connections with ACs in all 10 strata, whereas ACs with $\mathrm{ON}$ and OFF responses may make connections only with ACs in strata 1, 2, and 4-6. Although it is unclear how such an asymmetrical synaptic network is organized at the ultrastructural level, the logic circuit in Figure 5 begins to suggest some computational algorithms for amacrine-amacrine synaptic wiring in the inner retina.

\section{Morphological diversity and classification of retinal amacrine cells}

We have shown that ACs in the tiger salamander, like their counterparts in other vertebrates, exhibit extreme morphological diversity (Vaney, 1990; MacNeil and Masland, 1998; Masland, 2001). ACs differ from one another in their shape and size of cell bodies, dendritic width and thickness, patterns of dendritic branching, and level(s) of dendritic stratification in the IPL. Certain morphological features are closely correlated with different physiological responses (e.g., the levels of dendritic stratification in the IPL of narrowly monostratified cells closely correlate with the $\Delta I_{\mathrm{C}} \mathrm{ON}$ and OFF responses), whereas other morphological features are not [e.g., the bistratified cell in strata 3 and 8 (cell $f$ in Fig. 2) and the monostratified cell in stratum 7 (cell $c$ in Fig. 2) have very similar light responses]. In addition, some ACs with dendrites ramifying in the same strata exhibit very different light responses (e.g., cell 17 and cell 18 in Fig. 4, both with diffuse dendrites ramified in strata $1-10$, exhibit different $\Delta I_{C} \mathrm{~s}$ and $\left.\Delta I_{\mathrm{Cl}} \mathrm{s}\right)$.

From these results, it is evident that AC classification is a complex task. In addition to levels of dendritic stratification in the IPL and the polarity and kinetics of $\Delta I_{\mathrm{C}}$ and $\Delta I_{\mathrm{Cl}}$, there are many other morphological and physiological parameters that define $\mathrm{AC}$ types. These parameters include the receptive field size, presynaptic and postsynaptic partners, neurotransmitter contents, and patterns of dendritic branching of the cells. Each of these additional parameters may "lump" or "split" the various cell types set forth by the previous parameters (e.g., types based on the different levels of dendritic stratification in Fig. $1 B$ ) into a new set of AC types. For example, cell 13 and cell 20 in Figure 4 resemble the dopaminergic (tyrosine hydroxylase-positive) ACs, and cell 12 and cell 19 in Figure 4 and cell $g$ in Figure 2 resemble the serotonin cells (Watt et al., 1988; Watt, 1992). Therefore the additional parameter (neurotransmitter content) lumps cells 13 and 20 into one type and cells 12 and 19 into another type. However, cell types 1 and 2 (Fig. $1 B$ ) both contain narrow-, medium-, and wide-field cells; thus, the addition of receptive field size as a parameter splits cell types 1 and 2 into six types of ACs. Therefore, because of the extreme morphological diversity and complex physiological and neurochemical variations, classification of retinal ACs is highly dependent on the parameters chosen. It may be impractical to expect a simple universal classification scheme for such a diverse population of interneurons in the retina. It is perhaps more useful, instead, to determine computational rules for individual parameters, such as the layer-by-layer rule for the $\mathrm{ON}-\mathrm{OFF}$ bipolar cell inputs described in this article, 
and then integrate them into a comprehensive framework that process various attributes of visual images presented to the eyes.

\section{REFERENCES}

Ammermuller J, Kolb H (1995) The organization of the turtle inner retina. I. ON- and OFF-center pathways. J Comp Neurol 358:1-34.

Boycott BB, Wassle H (1991) Morphological classification of bipolar cells in the macaque monkey retina. Eur J Neurosci 361:1069-1088.

Boycott BB, Wassle H (1999) Parallel processing in the mammalian retina. Invest Ophthalmol Vis Sci 40:1313-1327.

Enroth-Cugell C, Robson JG (1966) The contrast sensitivity of retinal ganglion cells of the cat. J Physiol (Lond) 187:517-552

Euler T, Wassle H (1995) Immunocytochemical identification of cone bipolar cells in the rat retina. J Comp Neurol 361:461-478.

Famiglietti Jr EV, Kolb H (1976) Structural basis for ON-and OFFcenter responses in retinal ganglion cells. Science 194:193-195.

Gao F, Wu SM (1998) Characterization of spontaneous inhibitory synaptic currents in salamander retinal ganglion cells. J Neurophysiol 80:1752-1764.

Hensley SH, Yang XL, Wu SM (1993) Relative contribution of rod and cone inputs to bipolar cells and ganglion cells in the tiger salamander retina. J Neurophysiol 69:2086-2098.

Hubel DH, Livingstone MS (1987) Segregation of form, color, and stereopsis in primate area 18. J Neurosci 7:3378-3415.

Hubel DH, Wiesel TN (1968) Receptive fields and functional architecture of monkey striate cortex. J Physiol (Lond) 195:215-243.

Hubel DH, Wiesel TN (1969) Anatomical demonstration of columns in the monkey striate cortex. Nature 221:747-750.

Hubel DH, Wiesel TN (1977) Ferrier lecture. Functional architecture of macaque monkey visual cortex. Proc R Soc Lond B Biol Sci 198:1-59.

MacNeil MA, Masland RH (1998) Extreme diversity among amacrine cells: implications for function. Neuron 20:971-982.

MacNeil MA, Heussay JK, Dacheux RF, Raviola E, Masland RH (1999) The shapes and numbers of amacrine cells: matching of photofilled with Golgi-stained cells in the rabbit retina and comparison with other mammalian species. J Comp Neurol 413:305-326.

Marc RE, Liu WL (2000) Fundamental GABAergic amacrine cell circuitries in the retina: nested feedback, concatenated inhibition, and axosomatic synapses. J Comp Neurol 425:560-582.
Masland RH (2001) The fundamental plan of the retina. Nat Neurosci 4:877-886.

Miller RF, Dacheux RF (1983) Intracellular chloride in retinal neurons: measurement and meaning. Vision Res 23:399-411.

Nelson R, Famiglietti Jr EV, Kolb H (1978) Intracellular staining reveals different levels of stratification for on- and off-center ganglion cells in cat retina. J Neurophysiol 41:472-483.

Pang JJ, Gao F, Wu SM (2002) Relative contributions of bipolar cell and amacrine cell inputs to light responses of ON, OFF and ON-OFF ganglion cells. Vision Res 42:19-27.

Ramon y Cajal S (1893) La retine des vertebres. Cellule 9:119-257.

Roska B, Werblin FS (2001) Vertical interactions across ten parallel, stacked representations in the mammalian retina. Nature 410:583-587.

Tokheim RL (1994) Digital principles, Ed 3. New York: McGraw-Hill.

Vallerga S (1981) Physiological and morphological identification of amacrine cells in the retina of the larval tiger salamander. Vision Res 21:1307-1317.

Vaney DI (1990) The mosaic of amacrine cells in the mammalian retina. In: Progress in retinal research, Vol 9 (Osborne N, Chader J, eds), pp 49-100. Oxford: Pergamon.

Watt CB (1992) A double-label study demonstrating that all serotoninlike immunoreactive amacrine cells in the tiger salamander retina express GABA-like immunoreactivity. Brain Res 583:336-339.

Watt CB, Yang SZ, Lam DM, Wu SM (1988) Localization of tyrosinehydroxylase-like-immunoreactive amacrine cells in the larval tiger salamander retina. J Comp Neurol 272:114-126.

Werblin FS (1978) Transmission along and between rods in the tiger salamander retina. J Physiol (Lond) 280:449-470.

Wong-Riley MTT (1974) Synaptic organization of the inner plexiform layer in the retina of the tiger salamander. J Neurocytol 3:1-33.

Wu SM (1987) Synaptic connections between neurons in living slices of the larval tiger salamander retina. J Neurosci Methods 20:139-149.

Wu SM, Gao F, Maple BR (2000) Functional architecture of synapses in the inner retina: segregation of visual signals by stratification of bipolar cell axon terminals. J Neurosci 20:4462-4470.

Yang XL, Wu SM (1997) Response sensitivity and voltage gain of the rod- and cone-bipolar cell synapses in dark-adapted tiger salamander retina. J Neurophysiol 78:2662-2673.

Yang XL, Gao F, Wu SM (2002) Non-linear, high gain and sustainedto-transient signal transmission from rods to amacrine cells in darkadapted retina. J Physiol (Lond) 539:239-251. 